\title{
A Non-Invasive Portable XRF System for Cultural Heritage Analyses
}

\author{
Nicholas Barbi ${ }^{1}$, Roberto Alberti ${ }^{2}$, Luca Bombelli ${ }^{2}$ and Tommaso Frizzi ${ }^{2}$ \\ 1. PulseTor LLC, Pennington, New Jersey USA \\ 2. XGLab S.R.L., Milan, Italy
}

A portable non-contact XRF system, using an SDD with the CUBE preamplifier, based on CMOS technology and replacing the traditional JFET [1], has been optimized for Cultural Heritage applications.

The most significant advantage of CUBE technology is that its performance optimizes at short pulse processor peaking times relative to the JFET (Figure 1). This means that the specified resolution of the SDD will be maintained from low to very high count rates, resulting in improved sensitivity and precision for EDX systems used with either electron or X-ray excitation (EDS or XRF).

The complete XRF instrument, called ELIO, is shown with dimensions in Figure 2-left. It consists of an analysis head, comprising the SDD with CUBE, the X-ray tube and power supply, a camera for recording the sample image, and two laser beams for reproducibly positioning the stand-off of the head from the analysis point, allowing the analysis to be done without contacting the object. The analysis head is typically used on a tripod, although it can be used in an enclosed chamber. The head is mounted on transverse linear slides for fine $\mathrm{x}-\mathrm{y}$ positioning of the analysis point. The $\mathrm{z}$ position is adjustable by a geared column on the tripod.

The instrument uses an SDD with nominal active area of $25 \mathrm{~mm}^{2}$ and $135 \mathrm{eV}$ Resolution at Mn Ka using a Cube preamplifier. The specified resolution is attained at all count rates achievable by the instrument. The X-ray tube has $4 \mathrm{~W}$ power, with $10-50 \mathrm{kV}$ selectable in $1 \mathrm{kV}$ steps, and typically incorporates a $\mathrm{Rh}$ anode, although other targets are available. A primary beam X-ray filter set is provided to improve sensitivity in specific regions of the spectrum.

The coincidence of two laser beams, as illustrated in the schematic on Figure 2-right, defines the optimum stand-off position of the head. The combination of the laser beam positioning with CUBE preamplifier gives high analytical reproducibility in a portable instrument.

The integrated camera records an image of the region around the analysis point, as shown in Figure 3, in which two points on a gold leaf surface (ca. 1500) were analysed. The spectra indicated a significantly higher Fe:Au ratio in Point 1 compared to Point 2. However, the recorded optical images show that the region around Point 1 has apparently undergone more erosion than the region around Point 2, perhaps explaining the spectral differences.

The analyses of NIST standard bronzes containing 0.02 and $0.07 \% \mathrm{~Pb}$ and similar levels of $\mathrm{Sn}$ will be will be presented. Fundamental Parameters estimates of composition are reported. Light element performance using a He flush technique will be compared to electron beam microanalysis results. 
[1] L. Bombelli, C. Fiorini, T. Frizzi, R. Alberti, R. Quaglia "Nuclear Science Symposium Conference Record, 2012, N1-202

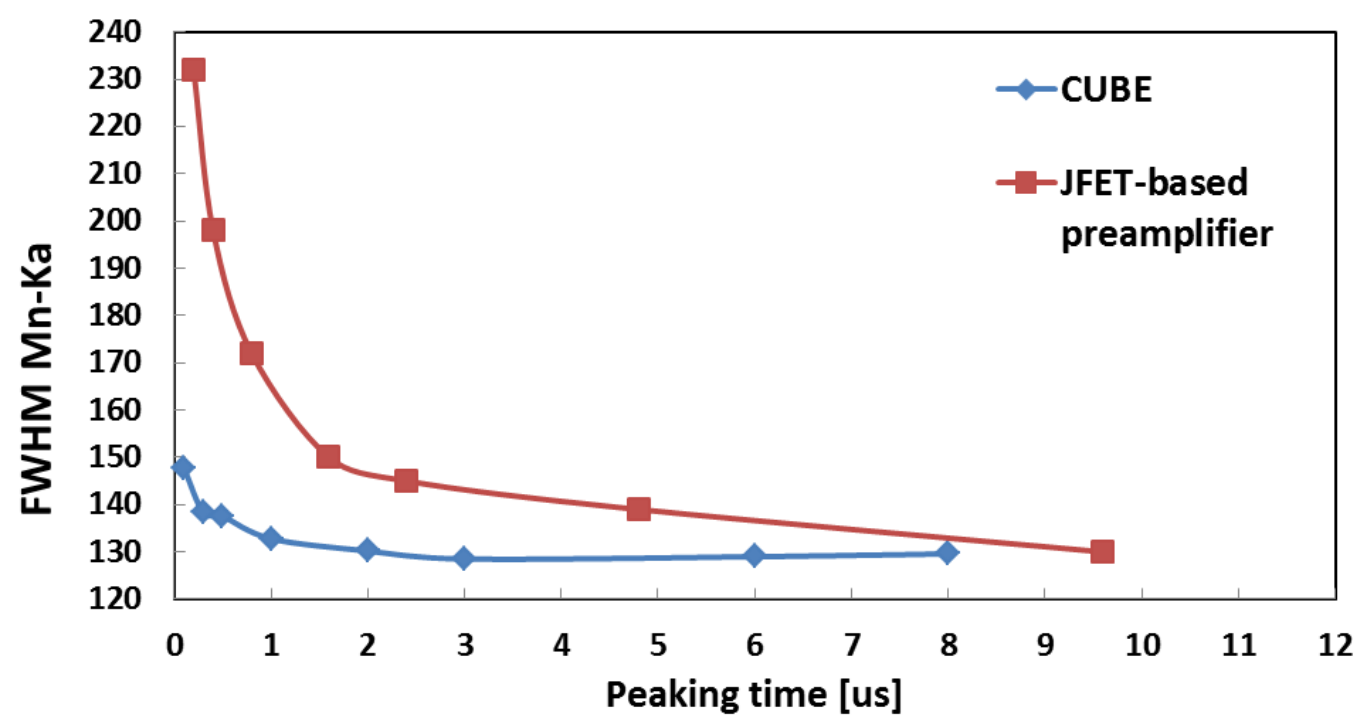

Figure 1: vata snowing the rsolution at the tast snaping times enabied by LUBE I ecnnolgy, meaning that high detector resolution will be maintained at high countrates.।

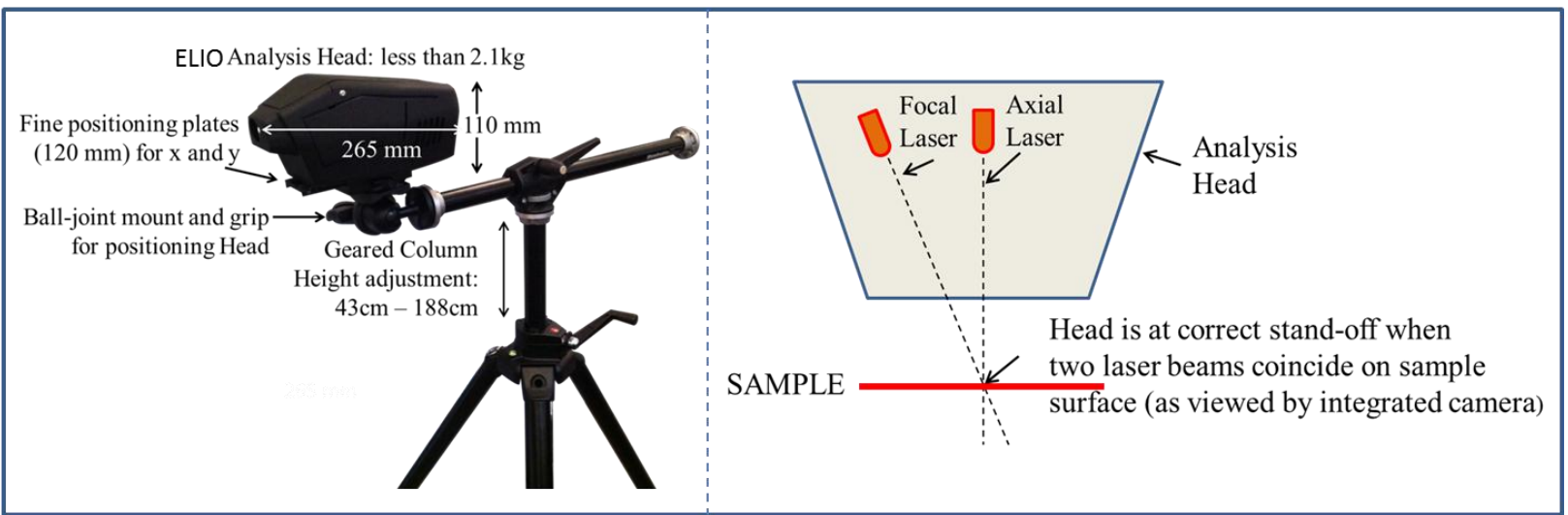

Figure 2 left: Dimensions and typical tripod mounting; right: dual laser method for positioning Head
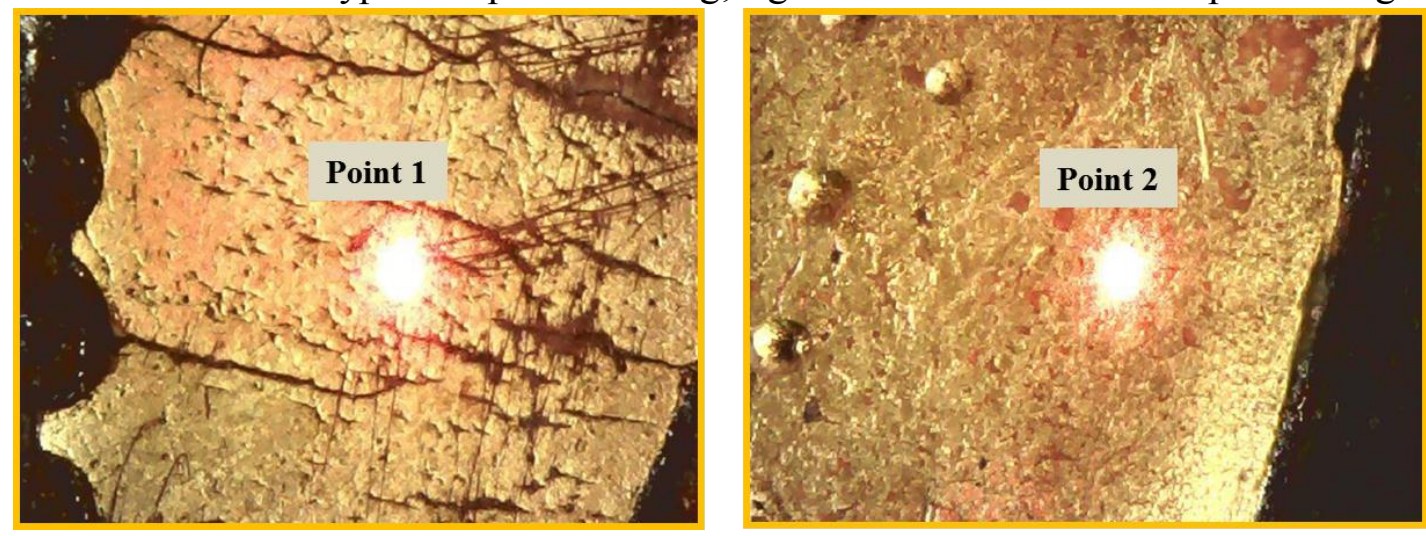

Figure 3: Two analysis points on gold leaf (ca. 1500). Integrated camera shows more erosion around Point 1 compared Point 2, explaining differences in Fe:Au ratios revealed in the corresponding spectra 